\title{
Deutsche Parteien in der Euro-Krise: Das Ende des Konsensprinzips?
}

\author{
Andreas Wimmel W $^{*}$
}

Kein Thema hat die deutsche Politik in den letzten Monaten so in Atem gehalten wie die ungelöste Euro-Krise. Immer umfangreichere Rettungspakete mussten geschnürt werden und immer größer wurden die Summen, die als Sicherheiten für überschuldete Mitgliedstaaten der Eurozone bereitgestellt werden sollen. Die Mitglieder des Deutschen Bundestages waren so häufig wie nie zuvor in der EU-Politik aufgerufen, sich in der öffentlichen Debatte zu positionieren und im Parlament über Gesetzentwürfe der christlich-liberalen Regierungskoalition zu europapolitischen Maßnahmen abzustimmen, die zuvor von den Staatsund Regierungschefs der Europäischen Union in Brüssel vereinbart worden waren. Diese Politisierung der europäischen Integration hat zur Entstehung neuer Konfliktlinien zwischen den (und innerhalb der) Bundestagsfraktionen geführt, die das zwischenparteiliche Konsensprinzip, das über Jahrzehnte die deutsche Europapolitik geprägt hatte, zumindest aufgeweicht haben. ${ }^{1}$ Während sich alle Parteien nach wie vor weitgehend einig waren, dass die Wirtschafts- und Währungsunion als ein Meilenstein des Integrationsprozesses nicht aufgegeben werden dürfe, herrschte große Uneinigkeit und Unsicherheit in der Frage, wie dieser Krise begegnet werden sollte und mit welchen Mitteln sie gelöst werden könnte.

Diese neuen Konfliktfelder in der Europapolitik sind nicht nur für die Struktur des deutschen Parteiensystems relevant, sondern beeinflussen ganz maßgeblich den praktischen Verhandlungsspielraum auf EU-Ebene. Das aktuelle Krisenmanagement wird in weiten Teilen von der deutschen und der französischen Regierung erarbeitet und vorangetrieben - alle Beschlüsse, die heute im Europäischen Rat getroffen werden, beruhen letztlich auf dem kleinsten gemeinsamen Nenner dieser beiden wirtschaftsstärksten EU-Mitgliedstaaten. ${ }^{2} \mathrm{Ob}$ und wie die Euro-Krise gelöst werden kann, liegt nicht in den Händen der EU-Institutionen, sondern wird in Berlin und Paris entschieden. ${ }^{3}$ Insofern ist die zukünftige Entwicklung der Europäischen Union und ganz besonders der Wirtschafts- und Währungsunion auch von innenpolitischen Konstellationen abhängig, da keine Regierung in einer so wichtigen europapolitischen Frage langfristig gegen alle Oppositionsparteien anregieren kann, sondern zumindest einen Grundkonsens anstreben muss. Ansonsten wäre die Verlässlichkeit von EUBeschlüssen massiv gefährdet, wenn nach Regierungswechseln in der nationalen Politik plötzlich eine ganz andere Linie in der Europapolitik vertreten wird und möglicherweise

* Dr. Andreas Wimmel, Institut für Politikwissenschaft, Universität Innsbruck.

1 Vgl. zur Geschichte der deutschen Europapolitik Gisela Müller-Brandeck-Bocquet/Corina Schukraft/Nicole Leuchtweis/Ulrike Keßler: Deutsche Europapolitik. Von Adenauer bis Merkel, Wiesbaden 2010; Markus Jachtenfuchs: Die Konstruktion Europas. Verfassungsideen und institutionelle Entwicklung, Baden-Baden 2002; Heinrich Schneider/Mathias Jopp/Uwe Schmalz (Hrsg.): Eine neue deutsche Europapolitik? Rahmenbedingungen - Problemfelder - Optionen, Bonn 2001.

2 Pascal Kauffmann/Henrik Uterwedde: Verlorene Konvergenz? Deutschland, Frankreich und die Euro-Krise, in: Aus Politik und Zeitgeschichte 43/2010, S. 13-19.

3 Am 2. Dezember 2011 hatten Merkel und Sarkozy eine Reform der EU-Verträge vorgeschlagen, um eine schärfere Aufsicht über die nationalen Haushalte der Euro-Staaten zu ermöglichen, die am Veto Großbritanniens gescheitert ist. Vgl. Frankfurter Allgemeine Zeitung: Berlin und Paris übernehmen die Führung, 3.12.2011 und Frankfurter Allgemeine Zeitung: Merkel und Sarkozy wollen Vertragsänderung im März 2012, 6.12.2011. 
Entscheidungen der Vorgängerregierung korrigiert werden. Ein genaueres Verständnis der Konfliktlinien deutscher Parteien in der Euro-Krise wäre somit hilfreich, um den Spielraum für weitere Rettungsmaßnahmen und die Konsequenzen von Regierungswechseln für die Zukunft der europäischen Wirtschafts- und Währungsunion abschätzen zu können.

Dieser Aufsatz vergleicht die Situationsdeutungen, Problemdiagnosen und Lösungsstrategien der Regierungs- und Oppositionsparteien im Deutschen Bundestag von den Beschlüssen zum ersten Finanzhilfspaket für Griechenland bis zur Abstimmung über die Ausweitung des EFSF-Rettungsschirms am 29. September 2011. Nach einem kurzen Überblick über die bisherigen Maßnahmen, die auf europäischer Ebene getroffen wurden, folgt im zweiten Abschnitt zunächst eine Aufstellung der Regierungserklärungen, Beratungen und Abstimmungen zur Lösung der Euro-Krise im Deutschen Bundestag vom 1. Januar 2010 bis zum 31. Dezember 2011. Im dritten Abschnitt werden dann die Positionen und Argumente der fünf Bundestagsfraktionen in den drei bis dato wichtigsten Parlamentsdebatten zur EuroKrise analysiert und gegenübergestellt. Das Fazit fasst die zentralen Ergebnisse zusammen und balanciert das Verhältnis von Konsens und Konflikt zwischen den deutschen Parteien in der Euro-Krise aus.

\section{Stationen der Euro-Krise: Vom Hilfspaket für Griechenland bis zum Europäischen Stabilitätsmechanismus (EMS)}

Hinter dem Schlagwort ,Euro-Krise“ verbirgt sich gleich eine ganze Reihe von wirtschafts- und finanzpolitischen Problemen in der Eurozone, die eng mit der hohen Staatsverschuldung einiger EU-Mitgliedstaaten verbunden sind und nicht isoliert voneinander betrachtet werden können. ${ }^{4}$ Nicht nur in der praktischen Politik, sondern auch in der Wirtschafts- und Finanzwissenschaft sind die Ursachen der Krise und mögliche Lösungsansätze heftig umstritten, weil gesicherte Erfahrungswerte unter den gegebenen Rahmenbedingungen weitgehend fehlen. Seit Anfang 2010 wurden diverse finanzpolitische Maßnahmen und institutionelle Reformen auf EU-Ebene umgesetzt, um die Wirtschafts- und Währungskrise in der Eurozone in den Griff zu bekommen. ${ }^{5}$

Die erste Maßnahme der Euro-Staaten in diesem Kontext war die Einrichtung eines provisorischen Stabilisierungsmechanismus, der auf einer Sondersitzung der europäischen Finanzminister am 9./10. Mai 2010 beschlossen wurde. Wesentlicher Bestandteil dieses ersten ,Euro-Rettungsschirms ‘ war die Einrichtung einer Europäischen Finanzstabilisierungsfazilität (EFSF), einer Zweckgemeinschaft, die Anleihen aufnehmen und Kredite von bis zu 440 Milliarden Euro vergeben kann, für die die Mitgliedstaaten bis zu einem abgestuften Betrag haften. ${ }^{6}$ Diesem Beschluss auf europäischer Ebene waren bereits bilaterale Kreditgarantien der Euro-Staaten, der Europäischen Zentralbank sowie des Internationalen Währungsfonds (IWF) speziell für Griechenland in Höhe von insgesamt rund 110 Milliarden Euro vorausgegangen.

Als sich die Euro-Krise in den folgenden Monaten dennoch fortsetzte, verständigten sich die Staats- und Regierungschefs der 17 Euro-Staaten auf dem EU-Gipfel am 16./17. De-

4 Martin Seidel: Aktuelle Probleme der europäischen Währungsunion, in: integration 4/2010, S. 334-349; Guy Kirsch: Die Euro-Krise ist (nicht nur) eine Währungskrise, in: Aus Politik und Zeitgeschichte 43/2010, S. 3-7.

5 Siehe aktuell Tobias Kunstein/Wolfgang Wessels: Die Europäische Union in der Währungskrise: Eckdaten und Schlüsselentscheidungen, in: integration 4/2011, S. 308-322.

6 Daniel Thym: Euro-Rettungsschirm: zwischenstaatliche Rechtskonstruktion und verfassungsgerichtliche Kontrolle, in: Europäische Zeitschrift für Wirtschaftsrecht (EuZW) 5/2011, S. 167-171, hier S. 168. 
zember 2010 auf die Schaffung eines dauerhaften Europäischen Stabilitätsmechanismus (ESM), der die zeitlich befristete EFSF ersetzen soll und dessen inhaltliche Ausgestaltung am 21. März 2011 vom Europäischen Rat festgelegt wurde. Neben einer Ausweitung des Kreditvolumens auf zunächst insgesamt 700 Milliarden Euro ist die Einführung eines ESMFonds vorgesehen, in den die Mitgliedstaaten 80 Milliarden Euro als Grundkapital direkt einbezahlen sollen. Der ESM soll dann, mit der EFSF vergleichbar, eigene Anleihen bis zu einer Höhe von 500 Milliarden Euro vergeben können. ${ }^{7}$

Das zweite Rettungspaket für Griechenland, das am 21. Juli 2011 von den Euro-Staaten beschlossen wurde, enthielt einige Reformen der inzwischen eingerichteten EFSF. Neben der freiwilligen Beteiligung des privaten Bankensektors an der Euro-Krise, unter anderem durch einen Anleihentausch, soll die EFSF zukünftig direkt Staatsanleihen angeschlagener Euro-Staaten ankaufen können, wenn die betroffenen Staaten bereit sind, ein tragfähiges Spar- und Reformprogramm umzusetzen. Zudem wurde vereinbart, dass die EFSF Kredite an andere Euro-Staaten mit längeren Laufzeiten und niedrigeren Zinsen ausstatten kann. ${ }^{8}$ Als zusätzliche Maßnahmen zur Beruhigung der Finanzmärkte haben die Staats- und Regierungschefs der Euro-Staaten am 26. Oktober 2011 einen Schuldenschnitt für Griechenland, eine Rekapitalisierung der daran beteiligten Banken sowie eine erneute Ausweitung des Krisenfonds EFSF auf rund eine Billion Euro in Aussicht gestellt. ${ }^{9}$

Mit den jüngsten Beschlüssen des EU-Gipfels vom 8./9. Dezember 2011 soll der Weg geebnet werden für eine Stabilitäts- und Fiskalunion, die die Euro-Staaten und andere EUMitgliedstaaten zu einer stärkeren Haushaltsdisziplin verpflichten soll. Bis März 2012 soll ein zwischenstaatlicher Vertrag unterzeichnet werden, in dem sich die teilnehmenden Staaten verpflichten, eine nationale Schuldenbremse einzuführen, mit der das jährliche strukturelle Staatsdefizit auf 0,5 Prozent des Bruttoinlandsprodukts (BIP) begrenzt wird. Die Einhaltung der Schuldenbremse soll von der EU-Kommission beaufsichtigt und voraussichtlich vom Europäischen Gerichtshof überprüft werden können. Ferner soll festgeschrieben werden, dass ein Staat mit einem Haushaltsdefizit von mehr als drei Prozent des BIP ein Defizitverfahren nur noch dann verhindern kann, wenn eine qualifizierte Mehrheit der Euro-Staaten dagegen stimmt. Schließlich soll der ESM bereits im Juli 2012, also ein Jahr früher als geplant, eingesetzt werden, und die Euro-Gruppe wird über weitere Hilfsaktionen aus dem Krisenfonds künftig nicht mehr einstimmig, sondern mit einer qualifizierten Mehrheit von 85 Prozent entscheiden. Die von der EU-Kommission vorgeschlagenen Euro-Bonds werden in der Schlusserklärung nicht berücksichtigt. ${ }^{10}$

7 Ulrich Hufeld: Zwischen Notrettung und Rütlischwur: der Umbau der Wirtschafts- und Währungsunion in der Krise, in: integration 2/2011, S. 117-131, hier S. 124.

8 Vgl. ausführlich Ansgar Belke/Christian Dreger: Das zweite Rettungspaket für Griechenland und Perspektiven für die Europäische Zentralbank, in: integration 3/2011, S. 214-227.

9 Frankfurter Allgemeine Zeitung: EU-Gipfel einigt sich auf Rekapitalisierung von Banken, 27.10.2011; von dem Vorhaben einer Umschuldung oder eines Schuldenschnitts für Griechenland hatte die Euro-Gruppe auf dem EU-Gipfeltreffen am 8./9. Dezember 2011 vorerst wieder Abstand genommen.

10 Vgl. Frankfurter Allgemeine Zeitung: Merkel: Gipfel ist der Durchbruch zu einer Stabilitätsunion und Mehr Haushaltsaufsicht für 26 EU-Länder, 10.12.2011. 


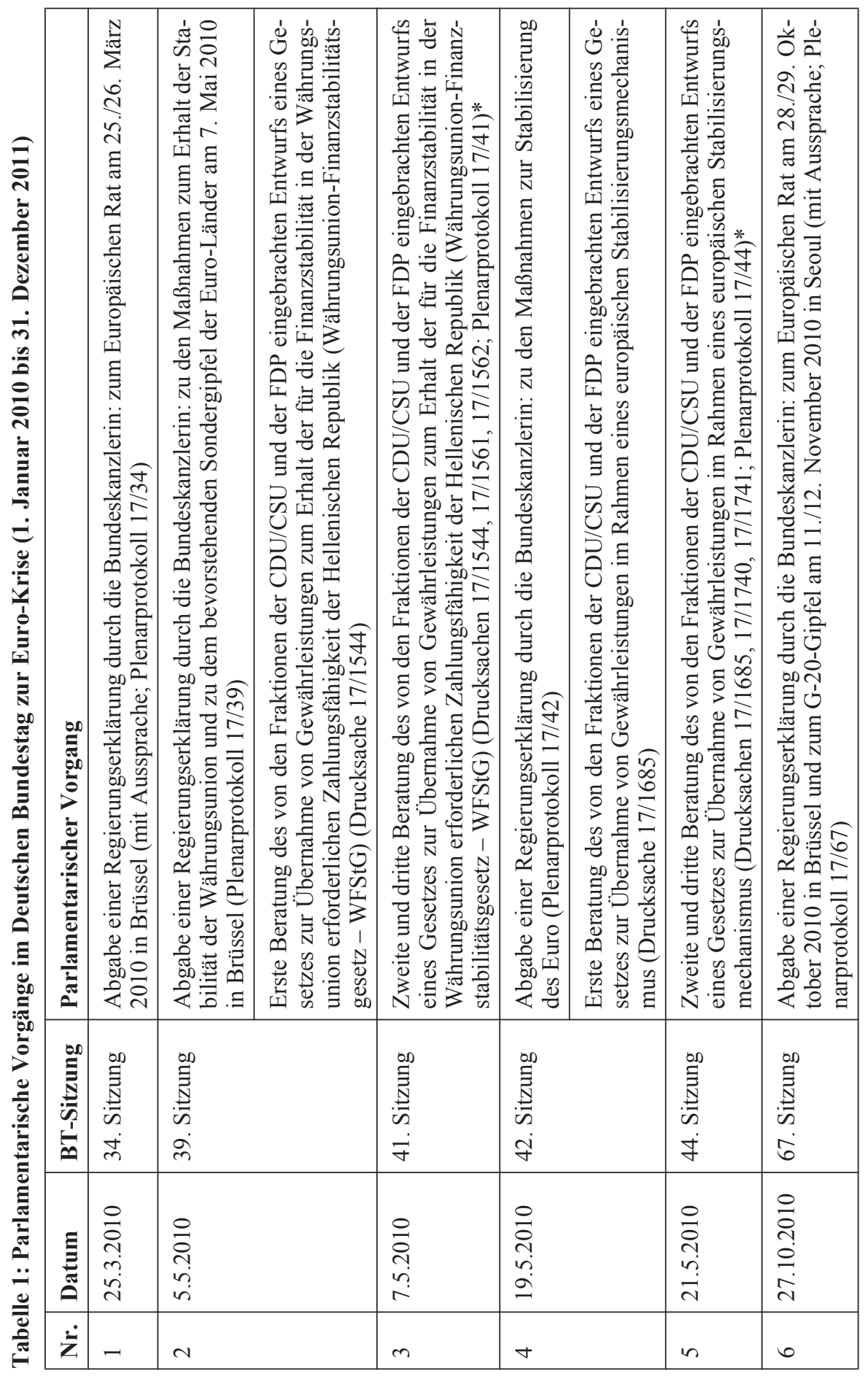




\begin{tabular}{|c|c|c|c|c|c|c|c|c|c|c|}
\hline 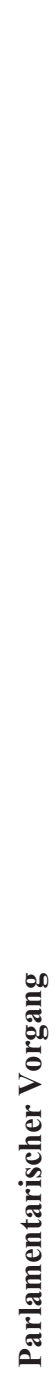 & 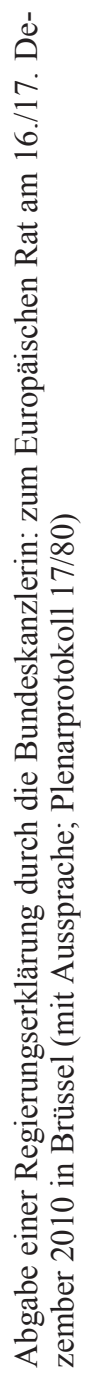 & 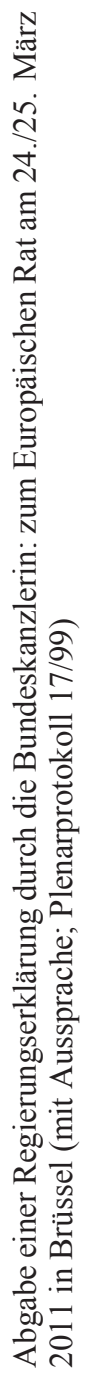 & 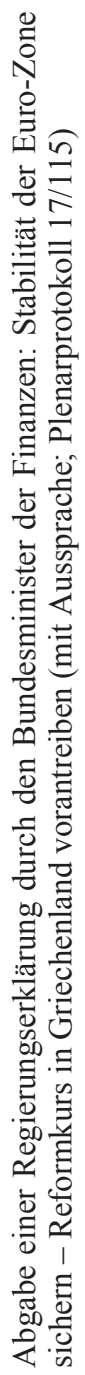 & 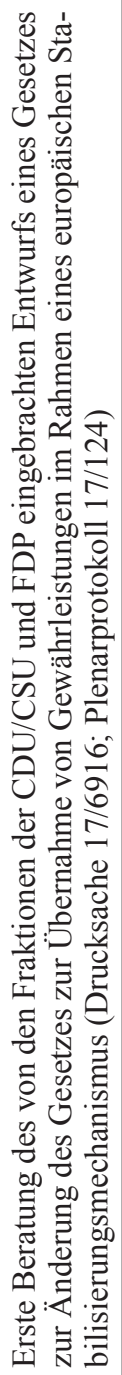 & 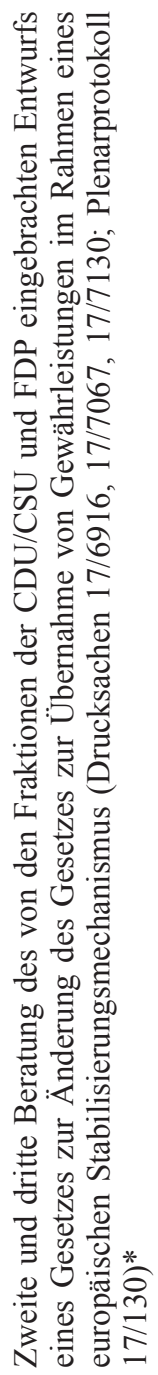 & 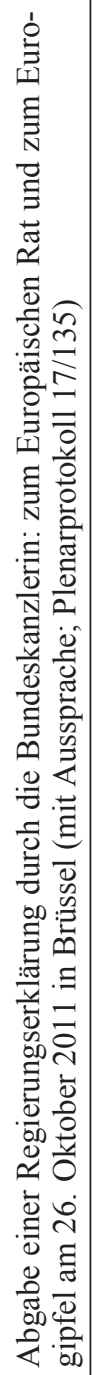 & 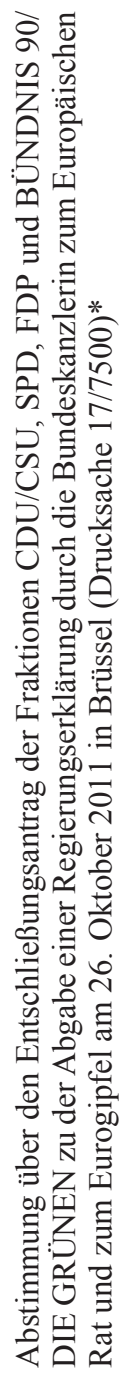 & 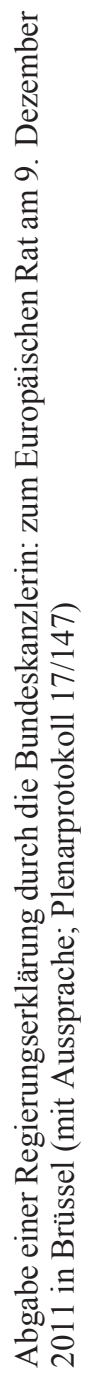 & 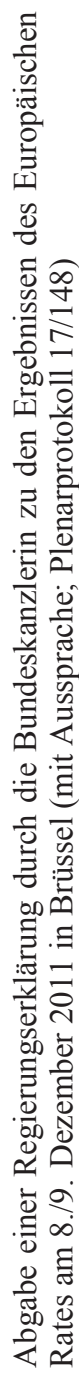 & 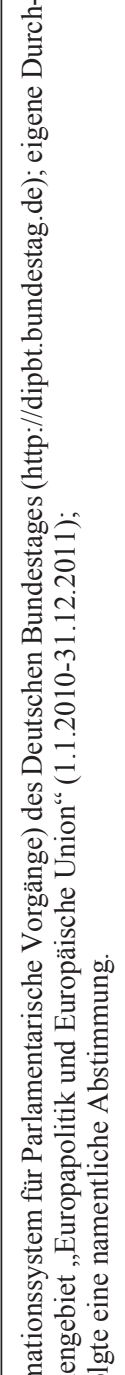 \\
\hline 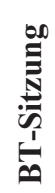 & 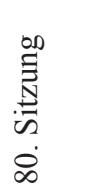 & 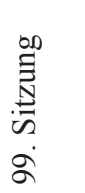 & 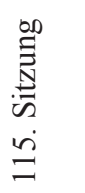 & 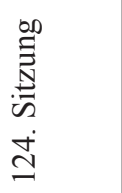 & 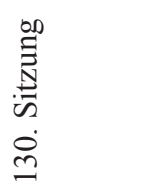 & 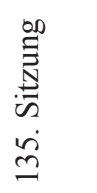 & & 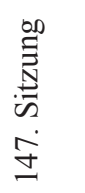 & 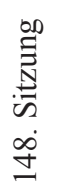 & 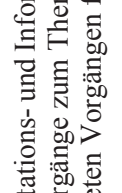 \\
\hline $\begin{array}{l}\text { E } \\
\text { E } \\
\text { Ẽ }\end{array}$ & 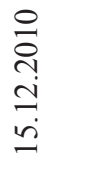 & 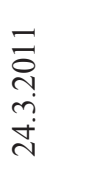 & $\begin{array}{l}\overline{0} \\
\text { กุ } \\
\stackrel{0}{0} \\
0\end{array}$ & $\begin{array}{l}\vec{\curvearrowright} \\
\stackrel{\sim}{\infty} \\
\infty\end{array}$ & 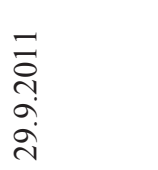 & 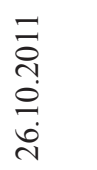 & & $\begin{array}{c}\stackrel{\sim}{u} \\
\stackrel{i}{i}\end{array}$ & 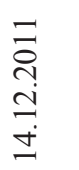 & $E \approx$ \\
\hline$\dot{z}$ & $r$ & $\infty$ & $a$ & $\stackrel{0}{-}$ & $=$ & $\simeq$ & & $\stackrel{m}{=}$ & \pm & \\
\hline
\end{tabular}




\section{Regierungserklärungen, Beratungen und Abstimmungen im Deutschen Bundestag}

Nie zuvor stand ein Thema der europäischen Integration in so kurzen Zeitabständen so häufig auf der Tagesordnung des Deutschen Bundestages wie die Euro-Krise. Die Ursachen dafür sind einerseits die ständig neuen und erweiterten Beschlussvorlagen, die im Europäischen Rat vereinbart wurden, und andererseits die starken Mitwirkungsrechte des Bundestages, wenn durch Entscheidungen auf europäischer Ebene die Hoheit über das Haushaltsrecht berührt wird. ${ }^{11}$ Dass der Bundestag nicht nur Stellungnahmen zur Euro-Krisenpolitik der Regierung abgeben durfte, sondern die wichtigsten Beschlüsse zur namentlichen Abstimmung gestellt wurden (siehe unten), begründet sich mit dem Rechtscharakter der Rettungsmaßnahmen, die nicht auf Basis der EU-Verträge vereinbart wurden, sondern überwiegend auf völkerrechtlichen Verträgen und zwischenstaatlichen Abkommen beruhen. ${ }^{12}$ Während die EU-Kommission und das Europäische Parlament aus diesem Grund bislang kaum Akzente setzen konnten, hat der Bundestag in der Euro-Krise deutlich mehr Kontrollund Mitbestimmungsrechte als im ordentlichen EU-Gesetzgebungsverfahren, weil jede Änderung und Erweiterung der Euro-Rettungsschirme von den Abgeordneten per nationaler Gesetzgebung beschlossen werden muss. ${ }^{13}$

Von Januar 2010 bis Dezember 2011 haben 14 Plenarsitzungen stattgefunden, in denen Regierungserklärungen, Gesetzentwürfe und Anträge, die von der Bundesregierung zur Lösung der Euro-Krise eingebracht wurden, im Mittelpunkt standen (siehe Tabelle 1). Die Regierungserklärung zum Europäischen Rat am 25./26. März 2010 in Brüssel war die erste Bundestagsdebatte zur Situation in Griechenland und zu deren Folgen für die Eurozone überhaupt, die letzte folgte im Anschluss an die Erklärung der Bundeskanzlerin zu den Ergebnissen des Europäischen Rates am 8./9. Dezember 2011 in Brüssel. In allen 14 Sitzungen hat eine Aussprache stattgefunden, an der sich jeweils Sprecher aus den fünf Bundestagsfraktionen beteiligt haben. Im Zusammenhang mit diesen parlamentarischen Vorgängen wurden zudem diverse Anfragen und Entschließungsanträge von Oppositionsparteien, insbesondere der Partei Die Linke (Linkspartei), eingebracht und teilweise zur Abstimmung gestellt, die allesamt scheiterten und nicht gesondert aufgeführt werden.

Neben den bislang acht Regierungserklärungen durch Bundeskanzlerin Angela Merkel mit anschließender Aussprache sind vor allem die ersten und zweiten Beratungen zu Gesetzentwürfen und Entschließungsanträgen relevant, über die danach namentlich abgestimmt wurde. Viermal hat der Bundestag bislang sein Votum zum Krisenmanagement der christlich-liberalen Regierungskoalition abgegeben: Am 7. Mai 2010 stimmten die Abgeordneten über das Währungsunion-Finanzstabilitätsgesetz (WFStG) ${ }^{14} \mathrm{ab}$, das den deutschen Beitrag zum ersten Hilfspaket für Griechenland festschreibt und mit dem die Regierung ermächtigt

11 Vgl. ausführlich zu den Mitbestimmungsrechten des Bundestages in EU-Angelegenheiten Timm Beichelt: Deutschland und Europa. Die Europäisierung des politischen Systems, Wiesbaden 2009, S. 245-278.

12 Siehe speziell zu den rechtlichen Grundlagen der Euro-Rettungsmaßnahmen Peter-Christian Müller-Graff: Euroraum-Budgethilfenpolitik im rechtlichen Neuland, in: integration 4/2011, S. 289-307.

13 Mit seinem Urteil vom 7. September 2011 zur Rechtmäßigkeit der Euro-Rettungsmaßnahmen hat das Bundesverfassungsgericht die Parlamentsrechte weiter gestärkt. Vgl. Bundesverfassungsgericht 2BvR 987/10, 2BvR 1485/10, 2BvR 1099/10. So müsse der Bundestag „die Kontrolle über grundlegende Entscheidungen der Haushaltspolitik behalten“, ferner dürfe die Budgetverantwortung „,nicht durch unbestimmte Ermächtigungen auf andere Akteure übertragen werden“. Vgl. Joachim Jahn: Keine unbestimmten Ermächtigungen, in: Frankfurter Allgemeine Zeitung, 8.9.2011.

14 Gesetz zur Übernahme von Gewährleistungen zum Erhalt der für die Finanzstabilität in der Währungsunion erforderlichen Zahlungsfähigkeit der Hellenischen Republik (Währungsunion-Finanzstabilitätsgesetz WFStG), in: Bundesgesetzblatt Teil I, Nr. 19 vom 7. Mai 2010, S. 537-538. 
wurde, für Kredite von bis zu 22,4 Milliarden Euro zu bürgen. Die nächste Abstimmung folgte nur zwei Wochen später am 21. Mai 2010 und betraf das Stabilisierungsmechanismusgesetz (StabMechG) ${ }^{15}$ zur Einrichtung eines provisorischen Euro-Rettungsschirms, den die EU-Finanzminister kurz zuvor am 9./10. Mai 2010 beschlossen hatten und mit dem die von Deutschland bereitgestellten Kreditgarantien für hoch verschuldete Euro-Staaten auf 123 Milliarden Euro erhöht wurden.

Die nächste namentliche Abstimmung, mit der das Stabilisierungsmechanismusgesetz vom 21. Mai 2010 geändert wurde, fand am 29. September 2011 statt. ${ }^{16}$ Die Gesetzesänderung betraf vor allem die Ausweitung der deutschen Haftungsgarantien für die EFSF auf nun 211 Milliarden Euro. Einen Monat später folgte die bislang letzte Abstimmung zur EuroKrise, als der Bundestag am 26. Oktober 2011 über einen gemeinsam von CDU/CSU, SPD, FDP und Bündnis 90/Die Grünen eingebrachten Entschließungsantrag ${ }^{17}$ entschied, mit dem sich Bundeskanzlerin Merkel ein parteiübergreifendes Mandat verschaffen wollte, um auf dem am gleichen Tag in Brüssel beginnenden EU-Gipfel weitere Maßnahmen zur Stärkung des Euro-Rettungsschirms verhandeln zu können. ${ }^{18}$

Tabelle 2: Ergebnisse der Abstimmungen des Deutschen Bundestages zur Euro-Krise (Ja/Nein/Enthaltung)

\begin{tabular}{|l|l|l|l|l|l|l|l|}
\hline Nr. & Datum & CDU/CSU & SPD & FDP & B'90/Grüne & Die Linke & Gesamt \\
\hline 1 & 7.5 .2010 & $234 / 4 / 0$ & $4 / 0 / 134$ & $92 / 1 / 0$ & $61 / 0 / 5$ & $0 / 67 / 0$ & $391 / 72 / 139$ \\
\hline 2 & 21.5 .2010 & $230 / 4 / 3$ & $0 / 1 / 128$ & $89 / 2 / 1$ & $0 / 0 / 63$ & $0 / 66 / 0$ & $319 / 73 / 195$ \\
\hline 3 & 29.9 .2011 & $226 / 10 / 1$ & $141 / 1 / 1$ & $89 / 3 / 1$ & $67 / 1 / 0$ & $0 / 70 / 0$ & $523 / 85 / 3$ \\
\hline 4 & 26.10 .2011 & $224 / 9 / 1$ & $129 / 4 / 3$ & $87 / 5 / 0$ & $63 / 1 / 0$ & $0 / 70 / 0$ & $503 / 89 / 4$ \\
\hline
\end{tabular}

Quelle: Eigene Auszählung auf Basis DIP (Dokumentations- und Informationssystem für Parlamentarische Vorgänge) des Deutschen Bundestages (http://dipbt.bundestag.de); endgültige Plenarprotokolle $17 / 41$ (7.5.2010), S. 4019 B-4022 C; $17 / 44$ (21.5.2010), S. 4443 A-4445 D; 17/130 (29.9.2011), S. 15234 A-15239 C; 17/135 (26.10.2011), S. 15978 D-15980 D.

Tabelle 2 zeigt die Ergebnisse dieser vier Abstimmungen im Deutschen Bundestag. Bei jeder Abstimmung konnte die sogenannte Kanzlermehrheit von 311 Stimmen erreicht werden, das heißt die christlich-liberale Koalition war nicht auf die Stimmen der Opposition

15 Gesetz zur Übernahme von Gewährleistungen im Rahmen eines europäischen Stabilisierungsmechanismus (Stabilisierungsmechanismusgesetz - StabMechG), in: Bundesgesetzblatt Teil I, Nr. 24 vom 22. Mai 2010, S. 627-628.

16 Gesetz zur Änderung des Gesetzes zur Übernahme von Gewährleistungen im Rahmen eines europäischen Stabilisierungsmechanismus, in: Bundesgesetzblatt Teil I, Nr. 51 vom 13. Oktober 2011, S. 1992-1994.

17 Entschließungsantrag der Fraktionen CDU/CSU, SPD, FDP und Bündnis 90/Die Grünen zu der Abgabe einer Regierungserklärung durch die Bundeskanzlerin zum Europäischen Rat und zum Eurogipfel am 26. Oktober 2011 in Brüssel, Bundestagsdrucksache 17/7500.

18 Zunächst war vorgesehen, nur den Haushaltsausschuss über die Leitlinien der Bundesregierung zum EU-Gipfel abstimmen zu lassen. Auf Drängen der Grünen-Fraktion wurde dann kurzfristig eine namentliche Abstimmung angesetzt, um dem Vorwurf zu entgehen, die Beteiligungsrechte des Bundestages seien verletzt worden. Vgl. Frankfurter Allgemeine Zeitung: Union vollzieht Kehrtwende/EFSF-Abstimmung nun doch im Plenum, 25.10.2011. 
angewiesen. ${ }^{19}$ Die CDU/CSU-Fraktion hat allen Entwürfen und Anträgen mit sehr deutlichen Mehrheiten zugestimmt, allerdings verweigerten bei den letzten beiden Abstimmungen zehn beziehungsweise neun Abgeordnete, etwa der euroskeptische CSU-Politiker Peter Gauweiler, die Gefolgschaft und stimmten mit Nein. Auch die FDP-Fraktion hat allen Bundestagsbeschlüssen zur Euro-Krise mit großen Mehrheiten zugestimmt, nur eine kleine Gruppe von Abweichlern formierte sich nach und nach um den Abgeordneten Frank Schäffler. ${ }^{20}$ Trotzdem konnten sich die Regierungsparteien bislang auf die Gefolgschaft von mehr als 90 Prozent ihrer Abgeordneten verlassen und das durchaus vorhandene innerparteiliche Konfliktpotenzial unter Kontrolle halten.

Der größte Schwenk im Abstimmungsverhalten im Verlauf der Euro-Krise war auf Seiten der SPD zu beobachten: Die SPD-Fraktion hat sich in den ersten beiden Abstimmungen fast einstimmig enthalten, erst die Entscheidungen im Jahr 2011 zur Ausweitung der deutschen Haftungsgarantien für die EFSF haben die Sozialdemokraten nahezu geschlossen unterstützt. Erst nachdem sie für ihre anfängliche Unentschlossenheit viel öffentliche Kritik einstecken musste, kündigte die SPD-Führung in einem Schreiben an die Bundeskanzlerin vor dem EUGipfel vom 21. Juli 2011 an, sich bei Abstimmungen zur Euro-Krise nicht länger enthalten, sondern die Hilfsmaßnahmen der Bundesregierung trotz weiterhin bestehender inhaltlicher Differenzen unterstützen zu wollen. ${ }^{21}$ Dennoch markieren diese ersten beiden Enthaltungen eine kleine Zäsur, weil die SPD in der Vergangenheit die allermeisten wichtigen EU-Entscheidungen, zum Beispiel den Vertrag von Maastricht, selbst dann mehrheitlich mitgetragen hat, wenn sie als Oppositionspartei mit bestimmten Aspekten der Europapolitik der Regierung unzufrieden war. ${ }^{22}$

Die Fraktion von Bündnis 90/Die Grünen hat dreimal die Politik der Bundesregierung in der Euro-Krise breit unterstützt, allein bei der Abstimmung über den Entwurf des Stabilisierungsmechanismusgesetzes vom 21. Mai 2010 enthielten sich die Grünen-Abgeordneten einstimmig. Die Linkspartei hat, entsprechend ihrer ablehnenden Grundhaltung gegenüber der wirtschaftsliberalen Stoßrichtung der deutschen Europapolitik, viermal geschlossen mit Nein gestimmt. ${ }^{23}$

19 Insbesondere bei den letzten beiden Abstimmungen war im Vorfeld unsicher, ob die ,Kanzlermehrheit‘ erreicht wird und welche politischen Konsequenzen gezogen werden müssten, sollte eine Stimmenmehrheit nur mit Unterstützung der Opposition zustande kommen. Vgl. Günter Bannas: Die diversen Mehrheiten der Frau Merkel, in: Frankfurter Allgemeine Zeitung, 28.9.2011.

20 In einer von Schäffler initiierten Mitgliederbefragung wurde die Einrichtung des dauerhaften Euro-Schutzschirms ESM und damit der europapolitische Kurs des FDP-Bundesvorstands von 54,5 Prozent der gültigen abgegebenen Stimmen unterstützt. Vgl. Frankfurter Allgemeine Zeitung: Knapper Sieg des FDP-Vorstands über Euro-Skeptiker, 17.12.2011.

21 Laut dem SPD-Parteivorsitzenden Sigmar Gabriel gelte diese Unterstützung selbst dann, wenn der Regierung eine eigene Mehrheit fehlen sollte. Vgl. Frankfurter Allgemeine Zeitung: SPD will Bundesregierung in der EuroKrise unterstützen, 19.7.2011.

22 Andreas Wimmel: Neue (alte) Konfliktlinien in der Europapolitik: Die Parlamentsdebatte zum Vertrag von Lissabon im Deutschen Bundestag, in: Zeitschrift für Parlamentsfragen 4/2009, S. 746-758.

23 Vgl. zur Linkspartei, die zwar nach eigener Aussage die Idee europäischer Integration nicht prinzipiell ablehnt, aber eine radikale Neuausrichtung europapolitischer Ziele und Inhalte fordert, Charles Lees: Limits of PartyBased Euroscepticism in Germany, in: Aleks Szczerbiak/Paul Taggart (Hrsg.): Opposing Europe? The Comparative Party Politics of Euroscepticism, Oxford 2008, S. 16-37. 


\section{Konfliktlinien in den Bundestagsdebatten zur Euro-Krise}

Diese Zahlen erlauben einen groben Überblick über die Positionen der deutschen Parteien und deren Abgeordneten zur aktuellen Krisenpolitik der Bundesregierung. Sie geben allerdings keine Auskunft über inhaltliche Konfliktlinien zwischen den Parteien, die auch dann bestehen können, wenn das Abstimmungsergebnis recht eindeutig ausfällt. Dass die Oppositionsparteien die Europapolitik der Regierung kritisieren (müssen), ist fast schon systemisch bedingt, allerdings ist die Spannbreite möglicher Kritikpunkte gerade in der EuroKrise breit gefächert. So könnte die Kritik der Opposition nur auf bestimmte politische Maßnahmen abzielen, während andere von ihr unterstützt werden, oder sie konzentriert sich mehr auf andere Aspekte, etwa das Zeitmanagement politischer Beschlüsse, die Führungsrolle Deutschlands in der Euro-Krise oder die Informationspolitik der Bundesregierung. Ferner kann nicht zwingend vorausgesetzt werden, dass die Regierungs- und die Oppositionsparteien als geschlossene Blöcke auftreten. So könnten auch innerhalb der politischen Lager Konfliktlinien sichtbar werden, die quer zur aktuellen Rollenverteilung im Parlament verlaufen.

In den folgenden Abschnitten wird das Verhältnis von Konsens und Konflikt zwischen den fünf Bundestagsfraktionen in der Euro-Krise aufgeschlüsselt. Im Mittelpunkt stehen Ähnlichkeiten und Unterschiede in den Situationsdeutungen, Problemdiagnosen und Lösungsstrategien der Parteien: Situationsdeutungen beschreiben den Status quo und betreffen die Frage, wie der aktuelle Krisenzustand entstehen konnte. Problemdiagnosen sind kritische Bewertungen aktueller politischer Entwicklungen und Entscheidungen, während Lösungsstrategien praktische Maßnahmen vorschlagen, wie bestimmte Mängel oder Defizite behoben werden können. Die vergleichende Inhaltsanalyse beschränkt sich auf diejenigen Parlamentsdebatten, denen namentliche Abstimmungen über Gesetzentwürfe zur Euro-Krise folgten: die Regierungserklärung und erste Beratung zum Währungsunion-Finanzstabilitätsgesetz vom 5. Mai 2010, ${ }^{24}$ die Regierungserklärung und erste Beratung zum Stabilisierungsmechanismusgesetz vom 19. Mai $2010^{25}$ sowie die erste und zweite Beratung zur Änderung des Stabilisierungsmechanismusgesetzes vom 8./29. September $2011 .{ }^{26}$ Für jede der Plenardebatten wurde der jeweils erste (und in der Regel ranghöchste) Sprecher jeder Partei ausgewählt. Die endgültigen Protokolle wurden mit Hilfe des Textanalyseprogramms MAXQDA analysiert. Dieses Programm erlaubt die geordnete Kategorisierung großer Textmengen, um für das Forschungsinteresse wichtige von weniger wichtigen Textpassagen zu trennen und diese auf Befehl gebündelt anzuzeigen, um sie besser auswerten zu können. Praktisch wurden zunächst alle Redebeiträge der Sprecher in das Programm importiert und dann die wichtigsten Abschnitte, die Auskunft über Gemeinsamkeiten und Differenzen zwischen den Parteien geben, markiert. Die dabei angewendete Methode lässt sich als computergestützte, qualitativ-hermeneutische Inhaltsanalyse bezeichnen. ${ }^{27}$

24 Deutscher Bundestag. Stenografischer Bericht. 39. Sitzung. Berlin, Mittwoch, den 5. Mai 2010. Plenarprotokoll 17/39, S. 3721 B-3745 A.

25 Deutscher Bundestag. Stenografischer Bericht. 42. Sitzung. Berlin, Mittwoch, den 19. Mai 2010, Plenarprotokoll 17/42, S. 4131 D-4157 B.

26 Deutscher Bundestag. Stenografischer Bericht, 124. Sitzung, Berlin, Donnerstag, den 8. September 2011. Plenarprotokoll 17/124, S. 14551 D-14576 D sowie Deutscher Bundestag. Stenografischer Bericht, 130. Sitzung, Berlin, Donnerstag, den 29. September 2011. Plenarprotokoll 17/130, S. 15204 A-15244 D.

27 Siehe dazu ausführlicher Udo Kuckartz: Einführung in die computergestützte Analyse qualitativer Daten, 3. Aufl., Wiesbaden 2010; Philipp Mayring: Qualitative Inhaltsanalyse. Grundlagen und Techniken, 10. Aufl., Weinheim/Basel 2009. 


\section{Die Bundestagsdebatte zum Finanzhilfspaket für Griechenland am 5./7. Mai 2010}

Bundeskanzlerin Angela Merkel rechtfertigte den Entwurf des Währungsunion-Finanzstabilitätsgesetzes mit dem Vorliegen einer „Notsituation“, die darin bestünde, dass „Griechenland faktisch keinen Zugang mehr zu den Finanzmärkten“ habe und daraus „Auswirkungen auf die Stabilität des Euro insgesamt" resultierten. In diesem Zusammenhang fiel auch der viel zitierte Ausspruch Merkels, die Hilfen für Griechenland seien ,alternativlos, um die Finanzstabilität des Euro-Gebietes zu sichern“. Im Mittelpunkt ihrer Regierungserklärung stand jedoch nicht zuerst die Verteidigung der Notwendigkeit der Kreditgarantien generell, sondern die Entkräftung des Vorwurfs, sie hätte viel zu spät auf die Euro-Krise reagiert und damit die Ausgangssituation noch verschlimmert. Merkel gab zu bedenken, dass zunächst die verfassungs- und vertragsrechtlichen Hürden und die genauen Voraussetzungen, unter denen Griechenland Finanzhilfen erhalte, geklärt werden mussten: „Ein guter Europäer ist nicht unbedingt der, der schnell hilft und damit vielleicht nur den Anschein erweckt, als ob er das Problem lösen würde“. Ferner kündigte Merkel diverse wirtschaftsund finanzpolitische Koordinierungen an, um die Eurozone dauerhaft zu stabilisieren, wandte sich aber gegen die Einführung einer Finanztransaktionssteuer, die im Vorfeld von der SPD-Führung als Teil einer gemeinsamen Resolution zur Euro-Krise gefordert wurde. ${ }^{28}$

Die damalige FDP-Fraktionsvorsitzende Birgit Homburger verteidigte ebenfalls die anfängliche Zurückhaltung der Bundesregierung, aber bezeichnete die Hilfen nun als Ultima Ratio, da sich „die Finanzmarktentwicklungen in Griechenland, wenn jetzt nicht gehandelt wird, auf die Finanzstabilität des Euro-Gebietes auswirkten“. Allerdings, so Homburger in Richtung der SPD-Kritiker, müsse man von einem Land, das in eine solche Situation kommt, zunächst einmal eigene Anstrengungen erwarten. Ansonsten hätte die Gefahr bestanden, dass „die Hilfen angenommen worden wären, dass aber keinerlei Sanierungsprogramm auf den Weg gebracht worden wäre“. ${ }^{29}$

Die Kritik von SPD und Bündnis 90/Die Grünen an der Politik der Bundesregierung konzentrierte sich in dieser frühen Phase der Euro-Krise vor allem auf zwei Punkte: Der SPDFraktionsvorsitzende Frank-Walter Steinmeier unterstrich in seiner Rede, dass man die Bundeskanzlerin nicht etwa für die deutsche Beteiligung am EU-Rettungspaket angreife, sondern im Gegenteil: „Wir werfen Ihnen vor, dass Sie erst jetzt handeln. Das Unheil, dass Sie bis hierhin angerichtet haben, ist nämlich gewaltig“. Die Bundesregierung habe „geschwankt wie ein Rohr im Wind“ und erkläre dieses Zögern „nachträglich zur Strategie“. Obwohl die Entstehung der Krise bereits vor Wochen und Monaten erkennbar gewesen wäre, habe sie die Dinge treiben lassen und rufe erst jetzt, wo es lichterloh brennt, nach der Feuerwehr: „Diese Regierung ist kein Brandschutz für Deutschland“. ${ }^{30}$ Jürgen Trittin beklagte ebenfalls die träge Reaktionszeit der Bundesregierung auf die Euro-Krise. So habe eine Anfrage bei dem Chef des Internationalen Währungsfonds (IWF), Strauss-Kahn, ergeben, dass ,wir über geringere Summen reden“w würden, ,wenn der IWF im Februar/März hätte tätig werden können“. 31

Der zentrale inhaltliche Kritikpunkt aller Oppositionsparteien, mit dem die SPD-Fraktion später ihre Stimmenthaltung begründete, richtete sich auf die Forderung nach einer Beteili-

28 Angela Merkel, in: Deutscher Bundestag. Plenarprotokoll 17/39, S. 3721 D-3727 A, hier S. 3721 D, S. 3724 C, S. 3723 B.

29 Birgit Homburger, in: ebenda, S. 3731 A-3733 B, hier S. 3731 C, S. 3731 D.

30 Frank-Walter Steinmeier, in: ebenda, S. 3727 B-3730 B, hier S. 3727 C, S. 3728 C.

31 Jürgen Trittin, in: ebenda, S. 3739 A-3741 B, hier S. 3740 A. 
gung der Banken und Finanzmärkte an den Kosten dieser Krise. Diese dürften „nicht wieder einseitig auf den Steuerzahler abgeladen werden“. ${ }^{32}$ Trittin forderte eine engere Koordination der Wirtschaftspolitik in der Europäischen Union und insbesondere die Einführung einer Finanztransaktionssteuer, um ,endlich dafür Sorge zu tragen, dass diejenigen, die mit Spekulationen Geschäfte machen, künftig auch für die Folgen dieser Spekulationen in Haftung genommen werden“. Zudem, so Trittin, wäre es fahrlässig gewesen, mit der Option des Ausschlusses Griechenlands aus der Eurozone euroskeptische Ressentiments zu schüren und den Griechen vorzuwerfen, sie hätten schlicht über ihre Verhältnisse gelebt. ${ }^{33}$ Gregor Gysi forderte für die Linkspartei einmal mehr die nachhaltige Regulierung und Besteuerung der Finanzmärkte und des Bankensektors, um die Ursachen solcher Krisen in den Griff zu bekommen, anstatt Milliardenhilfen zur Verfügung zu stellen, die den Bundeshaushalt auf Jahre hinaus belasteten. Solange diese Forderungen nicht erfüllt würden, „gibt es von uns auch keine Zustimmung für das Gesetz". ${ }^{34}$

Trotz dieser Oppositionskritik am Timing des Krisenmanagements der Bundesregierung war die erste Abstimmung zur Euro-Krise im Bundestag durch einen breiten Konsens getragen, dass Griechenland geholfen werden müsse, um die Währungsunion zu retten - auch oder gerade im deutschen Interesse. Die Enthaltung der SPD-Fraktion resultierte eher aus dem Scheitern eines gemeinsamen Entschließungsantrags zur Kontrolle der Finanzmärkte als aus einer grundsätzlichen Skepsis gegenüber dem Hilfspaket für Griechenland. ${ }^{35}$ Die Fraktion von Bündnis 90/Die Grünen stimmte zwar letztlich mit großer Mehrheit für den Gesetzentwurf, kritisierte aber die zu defensiven Maßnahmen der Regierungskoalition in einem Entschließungsantrag, der auch von der SPD-Fraktion unterstützt wurde. ${ }^{36}$ Nur die Linkspartei votierte geschlossen gegen die Hilfskredite für Griechenland, weil ihrer Auffassung nach viel weitreichendere und striktere Regulierungen notwendig wären, um den Finanzkapitalismus in geordnete Bahnen lenken zu können.

\section{Die Bundestagsdebatte zum Europäischen Stabilisierungsmechanismus am} 19./21. Mai 2010

Nur zwei Wochen später waren die Bundestagsabgeordneten aufgerufen, über die Einrichtung des provisorischen Stabilisierungsmechanismus und über eine Erhöhung der deutschen Kreditgarantien auf 123 Milliarden Euro abzustimmen. Bundeskanzlerin Merkel warb in ihrer Regierungserklärung vehement für die im Europäischen Rat beschlossene Ausweitung der Hilfsmaßnahmen: „Die Währungsunion ist eine Schicksalsgemeinschaft [...], denn scheitert der Euro, dann scheitert Europa. Wenden wir diese Gefahr aber ab, dann werden der Euro und Europa stärker als zuvor sein“. Im Gegensatz zu der ersten Abstimmung musste sie nun jedoch um die Annahme des Gesetzentwurfs bangen, da neben der SPD-Fraktion auch die Fraktion von Bündnis 90/Die Grünen angekündigt hatte, sich enthalten zu wollen, wenn ihre Forderungen erneut ignoriert würden. Und auch in den eigenen Reihen, vor allem unter den FDP-Abgeordneten, mehrten sich die Stimmen, denen dieses erweiterte Hilfspaket

32 Frank-Walter Steinmeier, in: ebenda, S. 3727 B-3730 B, hier S. 3729 D.

33 Jürgen Trittin, in: ebenda, S. 3739 A-3741 B, hier S. 3741 B, S. 3740 A-D.

34 Gregor Gysi, in: ebenda, S. 3733 B-3736 A, hier S. 3736 A.

35 Die gemeinsame Entschließung zur Einführung einer Transaktionssteuer, die die SPD als Voraussetzung für ihre Zustimmung formuliert hatte, scheiterte vor allem am Widerstand der FDP. Vgl. Frankfurter Allgemeine Zeitung: Wie ein Nebenthema den Konsens verhinderte, 7.5.2010.

36 Entschließungsantrag der Fraktion Bündnis 90/Die Grünen zu der dritten Beratung des Gesetzentwurfs der Fraktionen der CDU/CSU und FDP - Drucksachen 17/1544, 17/1561, 17/1562, Bundestagsdrucksache 17/1640. 
unverständlich war oder zu weit ging. ${ }^{37}$ Deutlicher als in ihrer letzten Regierungserklärung sprach sich Merkel für eine stärkere Regulierung der Finanzmärkte aus, die - auch wenn sie nicht allein für die Euro-Krise verantwortlich seien - wie „Brandbeschleuniger gewirkt“ hätten. Sie habe den Auftrag der Koalitionsfraktionen sehr wohl wahrgenommen, die sagten: „Wir brauchen eine Besteuerung der Finanzmärkte“. ${ }^{38}$

Für die FDP-Fraktion sprach abermals zuerst Birgit Homburger, die sich wie Merkel für die Annahme des Gesetzentwurfs einsetzte, nachdem sich die Euro-Krise weiter verschärft hatte. Auch Homburger legte in ihrer Rede etwas mehr Gewicht auf die „Regulierung der Finanzmärkte“ und forderte „die Beteiligung der Finanzmärkte an den Kosten der Krise“, gab allerdings zu bedenken, dass gegen die Spekulation mit Staatsanleihen nur europa- und weltweite Lösungen helfen würden. Schließlich entgegnete sie der Opposition, nicht zuerst die Finanzmärkte, sondern „die Tatsache, dass Staaten über ihre Verhältnisse gelebt haben“, sei ursächlich für die Euro-Krise. ${ }^{39}$

Frank-Walter Steinmeier, der wieder als erstes für die SPD-Fraktion sprach, reagierte erstaunt auf den Schwenk der Kanzlerin. Erst vor zwei Wochen habe Merkel die Forderungen der SPD nach einer Besteuerung der Finanzmärkte als naiv abgetan, nun sei schon zu befürchten, dass „Sie am Ende so tun werden, als wären sie von Ihnen erfunden worden“. Anstatt in der größten Krise seit dem Zweiten Weltkrieg Führungsstärke zu zeigen, wirke Merkel „wie eine Getriebene der Märkte [...], wie eine Getriebene von der FDP und am Ende sogar wie eine Getriebene von der eigenen Partei““. ${ }^{40}$ Auch Jürgen Trittin kritisierte für die Fraktion von Bündnis 90/Die Grünen den vermeintlichen Schlingerkurs der Kanzlerin bei der Regulierung und Besteuerung von Finanztransaktionen, um die Kosten der Hilfskredite auffangen zu können. ${ }^{41}$ Hinzu komme, dass die Bundesregierung das Parlament nicht ausreichend in Kenntnis setze, was in Brüssel eigentlich beschlossen werde: „Es ist immer dasselbe Muster: Entscheidungen verschleppen, hier im Bundestag verschleiern, was genau verhandelt wird. Keiner rückt mit der Sprache wirklich heraus“ “. ${ }^{42}$ Trittin ergänzte, dass bislang nicht mehr als eine unverbindliche Absichtserklärung vorliege, wie die Milliardenkredite verwaltet werden sollen, ohne den Bundestag über die Planungen zu informieren: „Das, was Sie uns mit diesem Vorschlag unterbreiten, ist eine Brüskierung des Bundestages““. 43

Steinmeier betonte, dass seine Fraktion die Erhöhung der Hilfskredite grundsätzlich unterstütze, es aber nicht bei einer reinen Kreditermächtigung bleiben könne. Wie schon vor der Abstimmung über das Währungsunion-Finanzstabilitätsgesetz machte er die Zustimmung seiner Partei von einer Reihe begleitender Beschlüsse zur Kontrolle und Beteiligung der Finanzmärkte abhängig, unter anderem der Einführung einer Finanztransaktionssteuer, die „schwarz auf weiß in einem Text stehen“ müssten: „Aber wie wir uns entscheiden, hängt davon ab, ob die heutigen Ankündigungen wirklich ernst gemeint sind und ob, wie Sie versprochen haben, den Worten tatsächlich Taten folgen“. 44 Als erneut keine Einigung erzielt werden konnte, wie die Milliardenkredite refinanziert werden sollen, enthielt sich die SPD-

37 Günter Bannas: Verwerfungen bis in die eigenen Reihen, in: Frankfurter Allgemeine Zeitung, 20.5.2010.

38 Angela Merkel, in: Deutscher Bundestag. Plenarprotokoll 17/42, S. 4125 D-4131 B, hier S. 4126 A-B, S. 4129 D, S. 4130 D.

39 Birgit Homburger, in: ebenda, S. 4135 A-4139 B, hier S. 4136 A-D, S. 4138 A.

40 Frank-Walter Steinmeier, in: ebenda, S. 4131 C-4135 A, hier S. 4131 D, S. 4132 B.

41 Jürgen Trittin, in: ebenda, S. 4144 C-4147 A, hier S. 4144 D.

42 Frank-Walter Steinmeier, in: ebenda, S. 4131 C-4135 A, hier S. 4132 C.

43 Jürgen Trittin, in: ebenda, S. 4144 C-4147 A, hier S. 4146 D.

44 Frank-Walter Steinmeier, in ebenda, S. 4131 C-4135 A, hier S. 4134 D-4135 A. 
Fraktion erneut und auch die Grünen-Abgeordneten verweigerten der Regierung diesmal ihre Zustimmung.

Die Konfliktlinien zwischen den politischen Lagern waren also weitgehend konstant geblieben: Griechenland muss geholfen werden, jedoch werden die von der Regierung vorgeschlagenen Mittel und Instrumente, um die Eurozone zu stabilisieren, von den Oppositionsparteien als zu zurückhaltend und zögerlich eingeschätzt. Neu war lediglich der Vorwurf, die Regierung betreibe undurchsichtige Arkanpolitik und verschweige den Bundestagsabgeordneten, in welcher Form und auf welchen Wegen die Vergabe der Milliardenkredite genau realisiert werden soll.

\section{Die Bundestagsdebatte zur Ausweitung der EFSF am 8./29. September 2011}

Die erste Beratung des Gesetzes zur Änderung des Stabilisierungsmechanismus, mit dem der deutsche Anteil an den EFSF-Bürgschaften auf 211 Milliarden Euro aufgestockt werden sollte, eröffnete Bundesfinanzminister Wolfgang Schäuble. Am Tag zuvor hatte schon Bundeskanzlerin Merkel die Erhöhung des Kreditvolumens im Rahmen der Plenardebatte zum Bundeshaushaltsplan 2012 verteidigt, sich aber vehement gegen die Einführung sogenannter Euro-Bonds ausgesprochen, also gemeinsamer Staatsanleihen mehrerer EU-Mitgliedstaaten, die dann kollektiv für die Rückzahlung dieser Schulden haften würden: „Euro-Bonds sind der Weg in die Schuldenunion“, so Merkel. ${ }^{45}$ Schäuble betonte insbesondere, dass Griechenland zwar einen Anspruch auf Solidarität habe, aber die nächste Kredittranche nur erhalte, wenn die vereinbarten Auflagen erfüllt würden, um die Defizite und die hohe Verschuldung zurückzuführen: „Die Anpassungsmaßnahmen können wir Griechenland nicht ersparen". 46

Für die FDP sprach der Fraktionsvorsitzende Rainer Brüderle, der ebenfalls den Druck auf Griechenland erhöhte: „Wenn die Griechen Zusagen nicht einhalten, gibt es kein Geld; das ist die Spielregel“". Die SPD-Fraktion kritisierte er für die Forderung nach Euro-Bonds, die eine „gesamtschuldnerische Haftung für alle europäischen Staatsschulden“ bedeuteten und seiner Auffassung nach nicht mit dem aktuellen Urteil des Bundesverfassungsgerichts zu den Beteiligungsrechten des Bundestages in Einklang zu bringen sei. Zudem, so Brüderle, wolle die SPD-Basis doch selbst keine Euro-Bonds: „Erklären Sie Ihren Arbeitnehmern doch einmal, welche Haftung wir mit einer solchen Wundertüte [...] eingehen würden“" ${ }^{47}$ In der dritten Beratung vom 29. September 2011 traf seine Kritik vor allem Jürgen Trittin, der nach ihm für die Fraktion von Bündnis 90/Die Grünen sprechen sollte: „Sie haben Deutschland das Dosenpfand beschert. [...] Wir werden verhindern, dass Sie Europa eine Blechwährung bescheren". 48

Die Hauptredner für die SPD-Fraktion waren in der ersten Beratung der Parteivorsitzende Sigmar Gabriel und in der zweiten Beratung der frühere Bundesfinanzminister Peer Steinbrück. Gabriel wiederholte einmal mehr die Kernkritik seiner Partei nicht an den Hilfsmaßnahmen allgemein, sondern an der verfehlten Politik der Zurückhaltung seitens der Regierung, durch die sich die ganze Krisensituation an den Finanzmärkten dramatisch verschlimmert hätte. Zudem entgegnete er der Bundeskanzlerin, Deutschland hafte auch ohne Euro-

\footnotetext{
45 Angela Merkel, in: Deutscher Bundestag. Stenografischer Bericht, 123. Sitzung, Berlin, Mittwoch, den 7. September 2011. Plenarprotokoll 17/123, S. 14469 D-14474 B, hier S. 14471 C.

46 Wolfgang Schäuble, in: Deutscher Bundestag. Plenarprotokoll 17/124, S. 14551 D-14554 C, hier S. 14553 A.

47 Rainer Brüderle, in: ebenda, S. 14560 A-14562 D, hier S. 14562 C, 14561 A.

48 Rainer Brüderle, in: Deutscher Bundestag. Plenarprotokoll 17/130, S. 15210 D-15213 C, hier S. 15211 D.
} 
Bonds längst für die „Schuldentitel der Krisenstaaten in Höhe von 120 Milliarden Euro, die die Europäische Zentralbank aufkaufen musste“, weil die Regierung diesen Ankauf durch die EFSF noch im März 2011 verhindert hätte: „Das sind die ersten Merkel-Bonds, die wir hier im Hause bekommen haben“. Mit dem vorliegenden Entwurf zur Erweiterung des Rettungsschirms ,schlagen Sie also das genaue Gegenteil von dem vor, was Sie vor wenigen Monaten verteufelt haben: den Ankauf von Schuldtiteln durch die EFSF“" ${ }^{49}$ Auch Steinbrück sah in der zweiten Beratung direkt vor der Abstimmung die Politik der Regierung in der Euro-Krise als gescheitert an: Griechenland werde aus eigener Kraft „,nicht mehr zu einigermaßen verträglichen Konditionen an die Kapitalmärkte zurückkehren können“" und wird „an einem Schuldenschnitt unter Einbeziehung der Gläubiger nicht vorbeikommen“. Die SPD habe seit ,anderthalb Jahren Vorschläge gemacht [...], wie eine umfassende und tiefgreifende Strategie zur Stabilisierung der Euro-Zone aussehen könnte“, ohne dass die Regierung ernsthaft das Gespräch gesucht hätte- Trotzdem, so Steinbrück, werde die SPD unbenommen ihrer grundsätzlichen Kritik am Krisenmanagement der Bundesregierung „aus einer übergeordneten Verantwortung für die Gesetzesänderung stimmen". ${ }^{50}$

Während der Vorsitzende der Linkspartei, Klaus Ernst, gegen die unsoziale Europapolitik der Regierung wetterte, da mit dem Gesetz nicht der Euro, sondern ,einzig und allein die Banken, die Versicherungskonzerne, die Hedgefonds und die Finanzinvestoren gerettet" würden, ${ }^{51}$ folgte Trittin der SPD-Linie. Der nun vorgesehene Ankauf von Staatsanleihen durch die EFSF sei eine erste Vergemeinschaftung von Schulden, welche die Regierung immer vermeiden wollte und damit ,die Unabhängigkeit der Europäischen Zentralbank auf schäbige Weise beschränkt" habe. Notwendig sei eine neue vertragliche Regelung, die eine „Koordination in der Steuerpolitik, in der Wirtschaftspolitik und bei den Sozialstandards“ festschreibt, um die Europäische Union auf eine „,neue Stufe der Vergemeinschaftung zu führen". 52

In den beiden Parlamentsdebatten vor der dritten Abstimmung zur Euro-Krise hat sich das Konfliktmuster zwischen Regierungs- und Oppositionsparteien weiter verfestigt: Die Sprecher der christlich-liberalen Koalition verteidigten die erweiterten Rettungsmaßnahmen, mussten sich aber von SPD und Grünen vorhalten lassen, nun endlich diejenigen Schritte umzusetzen, die die Opposition lange gefordert hatte, aber von der Regierung zurückgewiesen wurden. Eine neue Dimension des zwischenparteilichen Streits war die Deutung über den Inhalt und die Folgen der EFSF-Reformen: Während die Koalition bestritt, dass die neuen Regelungen ihren früheren Aussagen widersprechen würden, argumentierte die rotgrüne Opposition, die Regierung wäre eingeknickt und müsste nun eingestehen, dass sie mit ihrer anfänglichen Zurückhaltung einen folgenschweren Fehler begangen habe.

\section{Fazit: Deutsche Parteien zwischen Konsens und Konflikt in der Euro-Krise}

Selbst wenn in der Summe der Konsens zwischen den Bundestagsparteien in der EuroKrise überwiegt, waren europapolitische Grundsatzentscheidungen niemals zuvor in der Geschichte der deutschen Europapolitik durch ein so erhebliches Konfliktpotenzial gekenn-

49 Sigmar Gabriel, in: Deutscher Bundestag. Plenarprotokoll 17/124, S. 14554 D-14559 A, hier S. 14555 D, S. 14556 B.

50 Peer Steinbrück, in: Deutscher Bundestag. Plenarprotokoll 17/130, S. 15206 C-15210 D, hier S. 15208 C/D, S. $15209 \mathrm{~A} / \mathrm{C}$.

51 Klaus Ernst, in: Deutscher Bundestag. Plenarprotokoll 17/124, S. 14563 A-14565 A, hier S. 14563 A-B.

52 Jürgen Trittin, in: ebenda, S. 14565 A-14567 B, hier S. 14565 D, S. 14567 B. 
zeichnet. Einerseits besteht nach wie vor eine proeuropäische Grundausrichtung, die CDU/ CSU, FDP, SPD und inzwischen auch Bündnis 90/Die Grünen tief in den Leitlinien ihrer Parteien verankert haben. Alle Parteien waren sich von Anfang an in der Beurteilung einig, dass die Wirtschafts- und Währungsunion nicht aufgegeben werden dürfe und Deutschland als Profiteur des Euro eine besondere Verpflichtung zukomme, aktiv zur Lösung der Finanzkrise beizutragen. Und auch bei den Ursachen der Euro-Krise herrschte weitgehende Übereinstimmung: Die Schaffung eines gemeinsamen Währungssystems bei sehr unterschiedlichen und nicht vereinheitlichten Wirtschafts- und Sozialordnungen der Euro-Staaten war ein schwerer und folgenreicher Konstruktionsfehler - strittig war lediglich, welche der Vorgängerregierungen die größere Verantwortung dafür trage. So blieb sich allein die Linkspartei in allen Phasen der Euro-Krise ihrer Fundamentalopposition gegenüber der Europapolitik der Bundesregierung treu und bezeichnete sich in der Debatte vom 26. Oktober 2011 als einzig wahre oppositionelle Kraft in diesem Hause. ${ }^{53}$

Andererseits unterscheiden sich die Lösungsstrategien zwischen Regierung und Opposition in der Euro-Krise erheblich: Obwohl die Bundesrepublik aus Sicht vieler Bürger schon astronomische Kreditgarantien bereitgestellt hatte, schürten die Oppositionsparteien nicht etwa euroskeptische Ressentiments, indem sie gegen die ständige Ausweitung der Hilfsmaßnahmen protestierten, sondern forderten von Beginn an schnellere und umfassendere Reaktionen. Während CDU/CSU und FDP zunächst kein zu großes Risiko eingehen wollten und nur den unbedingt notwendigen nächsten Schritt einleiteten, hatten SPD und Bündnis 90/Die Grünen aus der Oppositionsrolle heraus leichtes Spiel, die Regierung für ihr vermeintliches Stückwerk vor sich herzutreiben. In weiser Voraussicht um die Ausmaße der Krise waren sie so der Koalition immer um eine Nasenlänge voraus. Natürlich ist es müßig darüber zu spekulieren, ob sich die Konfliktkonstellation in vertauschten Rollen anders dargestellt hätte, allerdings sind die Forderungen des linken Lagers nach mehr staatlichen Eingriffen und Regulierungen der EU-Finanzmärkte kein Phänomen, das erst mit der Euro-Krise zu Tage getreten ist. Schon in der Bundestagsdebatte zum Vertrag von Lissabon 2008 hatte die SPD-Fraktion zusammen mit Bündnis 90/Die Grünen zu einer moderaten Europaskepsis zurückgefunden und vor allem das Fehlen einer „sozialen Dimension“ der europäischen Integration beklagt. ${ }^{54}$

Unstrittig dürfte damit sein, dass der Handlungsspielraum der christlich-liberalen Koalition im weiteren Verlauf der Euro-Krise weder durch Proteste der Oppositionsparteien gegen zu hohe Milliardenkredite eingeschränkt werden wird, noch dass ein Regierungswechsel zu einer Kehrtwende in der Euro-Rettungspolitik Deutschlands führen könnte. Trotz der aktiven Bemühungen der Bundesregierung waren die Oppositionsparteien nicht etwa Störfaktoren, sondern vielmehr Antriebskräfte einer Ausweitung der Hilfsmaßnahmen und verstärkter Finanzmarktregulierungen. Im Gegensatz zu anderen Mitgliedstaaten der Eurozone wie zum Beispiel Österreich, in denen Parteien wie die FPÖ öffentlich Stimmung machen gegen Milliardenkredite an „EU-Pleitestaaten“, die zu einer „Ausplünderung“ österreichischer Steuerzahler führten, ${ }^{55}$ rufen SPD und Grüne nicht nach weniger, sondern nach mehr Integration.

53 Gregor Gysi, in: Deutscher Bundestag. Stenografischer Bericht, 135. Sitzung, Berlin, Mittwoch, den 26. Oktober 2011. Plenarprotokoll 17/135, S. 15960 D-15963 C, hier S. 15963 A.

54 Und das, obwohl die SPD damals nicht in der Opposition, sondern als Regierungspartner in der Großen Koalition für die Vertragsinhalte mitverantwortlich war, vgl. Andreas Wimmel/Erica E. Edwards: The Return of 'Social Europe'. Ideas and Positions of German Parties towards the Future of European Integration, in: German Politics 2/2011, S. 293-314.

55 Vgl. Der Standard: FPÖ gegen Unterstützung von ,EU-Pleitestaaten‘, 16.5.2011. 
Insofern können sich CDU/CSU und FDP relativ sicher sein, dass sie mit weiteren Hilfsmaßnahmen keine euroskeptische Stimmungsmache seitens des politischen Gegners provozieren. Und auch im Falle einer anders zusammengesetzten Bundesregierung steht nicht zu befürchten, dass Deutschland seine Kreditzusagen reduziert oder weitere Entscheidungen zur Stabilisierung der Eurozone blockiert, solange die drei Oppositionsparteien nicht einen Politikwechsel um 180 Grad vornehmen.

Trotzdem hat die Euro-Krise gerade die SPD in eine strategisch schwierige und undankbare Lage manövriert: Obwohl die Partei erhebliche Zweifel an der Wirksamkeit des Krisenmanagements der Regierung hatte, war sie mehr oder weniger gezwungen, die EuroBeschlüsse mitzutragen oder wenigstens nicht gegen sie zu stimmen, wollte sie nicht als euroskeptische Partei erscheinen und auf eine Stufe mit der Linkspartei gestellt werden. Steinmeier hat dieses Spannungsverhältnis in der Plenardebatte vom 26. Oktober 2011 implizit selbst eingestanden, als er die Kanzlerin fragte, ob sie verstünde, dass „es in meiner Fraktion nicht wenige gibt, die fragen: Warum sollen wir eigentlich für die [Regierung] die Kohlen aus dem Feuer holen, warum sollen wir für das Chaos, das die angerichtet haben, noch die Finger heben?" 56 Bislang haben die Sozialdemokraten dennoch an der Tradition festgehalten, bei Fragen der europäischen Integration mehr mitzuregieren als zu opponieren, auch wenn die Lösungsansätze zwischen den beiden politischen Lagern diesmal weiter auseinanderlagen als jemals zuvor in der Europapolitik. ${ }^{57}$

In letzter Konsequenz besteht das Konsensprinzip also weiter, auch wenn sich die inhaltlichen Schnittstellen zwischen den Regierungs- und Oppositionsparteien in der Euro-Krise deutlich verkleinert haben. Trotzdem kann heute mit einiger Sicherheit davon ausgegangen werden, dass Deutschland auch zukünftig massive Anstrengungen und Kosten auf sich nehmen wird, um die Wirtschafts- und Währungsunion zu stabilisieren - unabhängig davon, welche Parteien gerade die Regierung stellen. Die nächsten Monate und Jahre werden zeigen, wie lange der europapolitische Basiskonsens im deutschen Parteiensystem in diesen stürmischen Zeiten noch aufrechterhalten werden kann.

56 Frank-Walter Steinmeier, in: Deutscher Bundestag: Stenografischer Bericht, 135. Sitzung, Berlin, Mittwoch, den 26. Oktober 2011. Plenarprotokoll 17/135, S. 15955 C-15958 C, hier S. 15956 B.

57 Vgl. Majid Sattar: Mitregieren statt opponieren. Die SPD befindet sich in der Euro-Krise in einem Dilemma, in: Frankfurter Allgemeine Zeitung, 27.10.2011. 